\title{
Germanica
}

\section{Portrait de l'artiste en vieillard : la représentation de la vieillesse dans la poésie de Hans Magnus Enzensberger}

Portrait of the artist as an old man: the representation of old age in the poetry of Hans Magnus Enzensberger.

Porträt des Künstlers als alter Mann: die Darstellung des Alterns in Hans

Magnus Enzensbergers Lyrik

\section{Hélène Boursicaut}

\section{OpenEdition}

\section{Journals}

Édition électronique

URL : http://journals.openedition.org/germanica/1566

DOI : 10.4000/germanica. 1566

ISSN : 2107-0784

\section{Éditeur}

Université de Lille

\section{Édition imprimée}

Date de publication : 10 juillet 2012

Pagination : 79-94

ISBN : 9782913857292

ISSN : 0984-2632

\section{Référence électronique}

Hélène Boursicaut, « Portrait de l'artiste en vieillard : la représentation de la vieillesse dans la poésie de Hans Magnus Enzensberger », Germanica [En ligne], 50 | 2012, mis en ligne le 19 juillet 2012, consulté le 06 octobre 2020. URL : http://journals.openedition.org/germanica/1566 ; DOI : https://doi.org/ 10.4000/germanica. 1566

Ce document a été généré automatiquement le 6 octobre 2020.

(c) Tous droits réservés 


\section{Portrait de l'artiste en vieillard : la représentation de la vieillesse dans la poésie de Hans Magnus Enzensberger}

Portrait of the artist as an old man: the representation of old age in the poetry of Hans Magnus Enzensberger.

Porträt des Künstlers als alter Mann: die Darstellung des Alterns in Hans

Magnus Enzensbergers Lyrik

Hélène Boursicaut

« Der Körper altert, das Gehirn wird jünger./ Ein tragischer Widerspruch, mit dem/ sich die

Wissenschaft abfindet, ohne zu murren ${ }^{1}$."

1 Enzensberger et la vieillesse : le thème peut de prime abord paraître incongru sous la plume d'un poète qui, selon la formule consacrée d'Alfred Andersch, a fait irruption dans l'histoire de la littérature allemande contemporaine sous l'étiquette du «jeune homme en colère ${ }^{2} »$ : un jeune homme qui, de fait, part en croisade contre une société ouest-allemande selon lui sclérosée et incapable de répondre aux aspirations de la jeune génération de l'après-guerre, car composée d'êtres vieillis avant l'âge, qui « attendent, trépassés, l'heure du trépas ${ }^{3} »$.

2 À ceci près que si l'on se penche sur les trois premiers recueils de Hans Magnus Enzensberger, Défense des loups (Verteidigung der Wölfe, 1957), Parler allemand (Landessprache, 1960) et Écriture braille (Blindenschrift, 1964), sous un angle autre que purement politique, on s'aperçoit qu'ils sont déjà traversés par une conscience aiguë du temps qui passe. Dans son premier opus, le poète, pourtant tout juste âgé de vingt-huit ans, va même jusqu'à rédiger ses dernières volontés qui, il est vrai, se terminent de façon fort irrévérencieuse: «Pour ce qui concerne la résurrection de la chair et la vie éternelle,/ permettez que je m'en occupe moi-même :/ 
c'est mon affaire, pas vrai? adieu.// Dans ma table de nuit il reste quelques cigarettes 4 . » D'emblée, toute vie humaine lui apparaît comme en sursis, ce qui lui fait dire dans une pirouette : «plus longtemps que tout [...] durera l'homme :// Jusqu'à ce que/ celui qui dans les profondeurs se tient/ ait compté nos secondes/ de dix à zéro ${ }^{5}$. " Il n'est pas jusqu'à l'écriture qui ne soit soumise à l'inexorable loi de la fugacité, puisque le troisième recueil du jeune poète pourtant promis à un brillant avenir s'achève sur un jugement sceptique, sinon désabusé : « ombres sont mes œuvres ${ }^{6}$."

Lorsqu'après dix ans de silence, plus exactement dix années consacrées à d'autres activités et d'autres formes d'écriture, Enzensberger renoue avec le genre poétique, c'est pour proposer dans Mausolée (Mausoleum ${ }^{7}$, 1975) trente-sept portraits de créateurs au soir de leur vie, avant de méditer dans Le Naufrage du Titanic (Der Untergang der Titanic ${ }^{8}$, 1978) sur la perte des illusions et la faillite des utopies, comme si les années précédant la cinquantaine se prêtaient déjà au bilan. Mais c'est surtout La Furie du disparaître (Die Furie des Verschwindens, 1980) qui marque un tournant dans l'œuvre du poète : l'intérêt croissant pour les petites choses de la vie quotidienne va de pair avec l'apparition, entre autres figures anonymes, de silhouettes de vieillards. La vieillesse est entrée dans l'univers poétique de Enzensberger et elle prendra une place de plus en plus importante dans les recueils des années quatre-vingt-dix, Musique de l'avenir (Zukunftsmusik, 1991), Kiosque (Kiosk, 1995), Plus léger que l'air (Leichter als Luft, 1995), et a fortiori dans ceux des années deux mille, L'histoire des nuages (Die Geschichte der Wolken, 2003) et Rébus (Rebus, 2009). On peut du reste noter que, contrairement aux recueils précédents qui ne portent aucune dédicace, le poète dédie son dernier ouvrage à ses parents dont il indique très précisément les dates de naissance et de mort, comme s'il s'agissait aussi de faire de Rébus un tombeau poétique en leur honneur'.

4 Quel regard Enzensberger porte-t-il sur le phénomène du vieillissement, qu'il observe d'abord de l'extérieur chez les autres, puis, les années passant, de l'intérieur, autrement dit à partir de sa propre personne? Comment, alors qu'on avance en âge, prendre la mesure du temps qui passe, quel sens donner à une vie qui va inéluctablement vers son terme, quelle attitude adopter face à la perspective de la mort, est-il possible d'envisager l'idée d'un au-delà? Autant d'interrogations qui reviennent de façon obsédante dans les six recueils cités supra à travers lesquels se dessine peu à peu un portrait de l'artiste en vieillard.

\section{La vieillesse : une autre appréhension du temps}

5 Au fil des ans, Enzensberger prend conscience que «le temps se réduit de minute en minute $^{10}$ ». Dès lors, c'est toute la perception de ce dernier qui s'en trouve modifiée. Le présent que le poète, dans ses premiers recueils, scrutait sans concession dans l'espoir qu'il aboutisse un jour à un avenir meilleur semble s'être figé en une durée seulement négative, car immuable. C'est comme si désormais il ne restait plus qu'un entre-deux inconfortable entre un passé qui ne cesse de reculer, voire de s'évanouir dans la mémoire, et un futur hypothétique, à moins que le futur ne soit tout simplement devenu le présent. Comme le fait remarquer Jörg Lau dans la biographie qu'il a consacrée à Enzensberger, «[1]'horizon se rétrécit. L'avenir est désormais pensé comme un temps qui ne nous appartient pas, qui ne s'enquiert pas de nous, auquel nous 
n'avons pas droit ${ }^{11}$ ». L'image de l'homme vieilli se superpose à celle du jeune homme. Et le poète qui, dans sa jeunesse, avait le sentiment d'être un mort vivant parmi d'autres morts vivants, de constater lui-même : «Lorsque j'avais vingt-ans, il y a bien longtemps de cela,/ je me faisais l'effet d'être un mort sans avenir./ Maintenant, l'avenir est là ${ }^{12}$.»

6 Cet avenir qui s'est effacé au profit d'un présent ressenti comme envahissant, car il abolit toute notion de dynamisme temporel, pousse le poète dans ses retranchements et l'oblige pour ainsi dire à adopter une posture de défense. Face à un monde de plus en plus dominé par les nouvelles technologies, ce dernier revendique son droit à aller à contre-courant, comme dans le poème Vieux media (Altes Medium) où il se livre à une véritable défense et illustration de la chose écrite. Pour ce faire, il endosse l'habit du présentateur ou de l'animateur de télévision et fait en quelque sorte son show, ce qui, paradoxalement, suggère qu'il connaît bien les codes de cette nouvelle société qu'il refuse, afin de tenter de gagner à sa cause un public d'analphabètes :

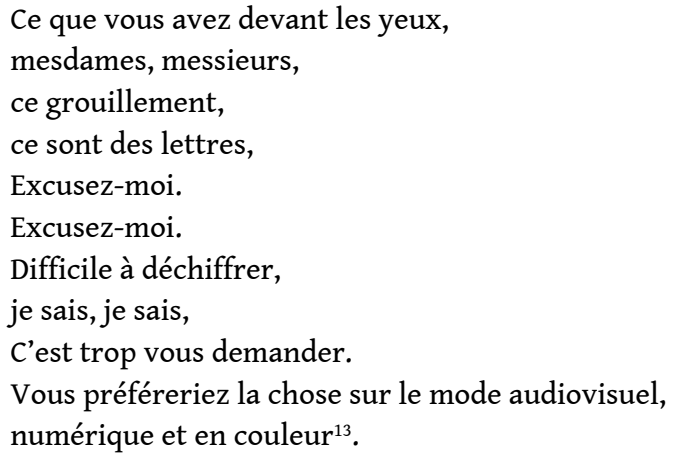

7 Feignant d'abord l'indulgence, multipliant même les courbettes, ce qui n'exclut pas du reste une certaine condescendance, le poète change pourtant radicalement d'attitude dans la deuxième strophe. Insérant dans son discours, mais sans guillemets, trois vers empruntés au panthéon littéraire allemand (Goethe, Gottfried Benn, Andreas Gryphius), il affirme haut et fort et sans se soucier le moins du monde de justifier son point de vue, le primat de la culture livresque, cette « virtual reality ${ }^{14}$ » d'une intensité bien autre que l'univers virtuel créé par les nouveaux medias : tant mieux pour ceux qui sauront décrypter les allusions, tant pis pour les autres. Comme il est loin, le temps où le poète, père et/ou maître, prônait « l'alphabétisation politique » de la jeunesse et conseillait à cette dernière de préférer les horaires de train et les cartes marines aux odes et aux chants ${ }^{15}$ ! Presque quarante ans plus tard et au risque de se faire accuser d'élitisme, il n'hésite pas à faire un pied de nez au " vulgum pecus » : «Je ne voulais pas vous froisser./ Mais vous savez bien ce que c'est:/ Certains sont incorrigibles ${ }^{16}$.» Affichant son opposition au jeunisme ambiant, synonyme à ses yeux d'inculture, le vieil impertinent se pose en garant de la tradition et assume ainsi pleinement ses réflexes réactionnaires au sens premier du terme, c'est-à-dire tournés vers le passé et hostiles à l'évolution des mœurs.

Cette attitude de défiance envers une modernité ressentie comme médiocre et vulgaire va de pair avec un net repli sur le passé. Plus on avance dans l'œuvre de Enzensberger, plus les textes se font intimistes, puisant aux sources de la matière biographique, c'està-dire l'enfance. Ces textes d'inspiration autobiographique ressuscitent des souvenirs paisibles, étroitement liés à des figures familiales qui tendent à remplacer les vieillards anonymes qu'on croisait au détour d'un vers dans La Furie du disparaître. Dans

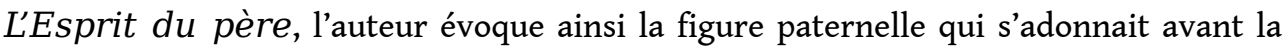


guerre à une étrange occupation consistant à imaginer des solutions pour améliorer le réseau ferroviaire allemand et passait des nuits entières à dessiner inlassablement des lignes de chemin de fer, souvenir qui inspirera du reste plus tard à Enzensberger le titre de sa première revue, Indicateur des chemins de fer (Kursbuch). Ce qui frappe dans ce portrait plein d'empathie et tout en nuances, c'est l'utilisation systématique du présent, comme si le défunt faisait à tout jamais partie intégrante de l'univers du fils vieillissant :

Certains soirs il est assis là,

comme autrefois, légèrement voûté,

fredonnant à sa table

sous la lampe de fer.

[...]

Parfois il m'écoute,

la tête baissée, la chevelure neigeuse,

sourit, absent, poursuivant

son projet merveilleux

que je n'arrive pas à comprendre,

qu'il ne terminera jamais.

Je l'entends fredonner ${ }^{17}$.

Autre évocation, cette fois avec une tendresse teintée d'humour, d'un personnage qui a également compté pour Enzensberger: le grand-père, patriarche vénéré qui, loin d'avoir fait vœu de tempérance, a su au contraire et en toute logique conjuguer bien vivre et bien mourir :

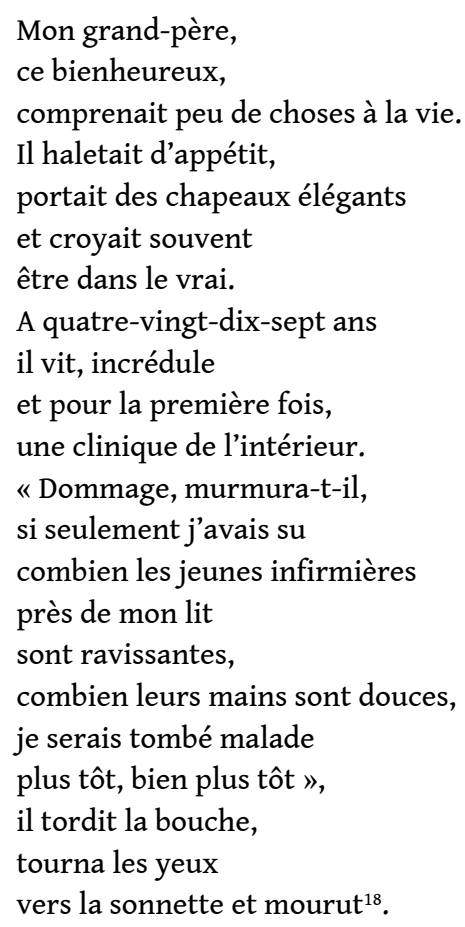

10 Toutefois, ce regard attendri sur les figures qui ont marqué l'enfance n'implique pas de regrets pour l'époque historique dans laquelle elle s'inscrit, car l'enfance du poète est également associée aux années du Troisième Reich et de la guerre. Dans Automne 44, Enzensberger se remémore certes sa fascination d'enfant inconscient, voyant "scintiller/ dans le ciel d'octobre sans nuages/ les vagues de bombardiers", ceux-ci fussent-ils en train de détruire sur leur passage les reliques familiales entassées au grenier, « les tasses de collection et les cheveux d'ange,/ les cartes postales parisiennes 
de grand-père/ (Oh là là) et sa boucle de ceinturon/ datant d'une autre guerre », mais c'est pour conclure avec la lucidité acquise grâce au recul par une formule laconique qui écarte toute velléité d'idéaliser le passé : «[...] dans la cave les cadavres, eux,/ sont toujours là̀ ${ }^{19}$. " Le poème Autrefois qui évoque très explicitement la société allemande des années trente sous le joug du national-socialisme démontre encore mieux que Enzensberger ne cède en rien à la tentation des images d'Épinal : "On n'imagine pas combien/ on se passe facilement/ du passé, pesant/ et sinistre. Aujourd'hui encore/ je ne sais pas exactement/ ce que c'est : la nostalgie. » Et pour ceux qui auraient encore des doutes, les derniers vers, multipliant les paradoxes, lèvent toute ambiguïté : « Rangez donc les vieilles photos./ Je préfère m'en remettre/ à l'éphémère./ Il ne laisse pas de place/ à l'émotion, est tenace/ et ne résiste devant rien ${ }^{20}$. » Preuve s'il en est que Enzensberger refuse de sacrifier aux sempiternelles considérations sur «le bon vieux temps » et ne verse pas dans le sentimentalisme, comme s'il tenait par là à se démarquer des travers souvent imputés aux gens âgés.

\section{Vieillesse et fin de vie}

11 L'appréhension différente de la dimension temporelle qui s'installe avec l'âge amène pour ainsi dire naturellement le poète à constater les ravages et les outrages du temps qui passe, moins du reste au niveau physiologique, comme si l'auteur répugnait à évoquer autrement que par simples petites touches la décrépitude physique, que sur le plan psychique et cognitif. Observant à une station de métro un couple âgé qui, sans un regard l'un pour l'autre, emprunte «l'escalator pour s'enfoncer dans l'Hadès quotidien ", Enzensberger tente de se représenter leur passé commun et remarque avec étonnement combien la passion s'est émoussée avec les années :

Ils ont pourtant un jour été tout feu tout flamme,

autrefois, je ne sais quand, perdus dans leurs pensées,

hors d'eux, rayonnants

de bonheur, ou non?

Comment en est-on arrivé là ? Depuis quand ? Et pourquoi ?

Dehors la neige, elle aussi, s'est une fois de plus

transformée en boue ${ }^{21}$.

De même que l'endroit public est assimilé à un lieu mythologique (les Enfers) et que la nature - ici la neige devenue boue - se fait le reflet de la désagrégation de l'amour, la vieillesse est associée à une sorte d'atonie émotionnelle. Mais plus encore que l'usure du corps et l'érosion des sentiments que Enzensberger note également chez lui ( «J'ai de plus en plus de mal à éprouver/ haine, envie, mépris,/ ces sentiments propres à la jeunesse./ Un signe de faiblesse $\left.{ }^{22} »\right)$, ce sont les défaillances du cerveau, en quelque sorte l'instrument de travail du poète et de l'intellectuel, qui inquiètent l'artiste en train de vieillir. Comme le fait remarquer Tae-Ho Kang dans sa thèse sur Enzensberger, "le phénomène de la 'fuite des idées', thématisé dans quelques-uns de ses poèmes, pourrait même à vrai dire être interprété comme un symptôme de vieillissement dont il fait pourtant une forme du dire poétique ${ }^{23}$. » Dans le recueil Kiosque, on trouve de fait une série de quatre textes intitulés Gedankenflucht, numérotés de I à IV et insérés dans chacune des quatre parties qui composent l'ouvrage. Ces poèmes qui font la part belle aux associations d'idées, aux digressions et au style " à sauts et à gambades » si cher à Montaigne, invitent évidemment à s'interroger sur le sens de cette écriture : 
s'agit-il pour le poète de rendre ainsi compte de son incapacité à trouver une cohérence à un monde de plus en plus chaotique ou le but est-il d'inventer une écriture de la vieillesse qui transcrirait, du moins en apparence, un laisser-aller conceptuel, un manque de rigueur, la fin des certitudes? Toujours est-il que la perte des facultés mentales constitue pour Enzensberger un réel motif d'angoisse. Lorsqu'il se penche sur le système limbique, région cérébrale souvent qualifiée de "cerveau de l'émotion » et qui joue un rôle crucial dans le processus de mémorisation, l'auteur ne se contente pas de disséquer cette énigme que constitue le cerveau avec une rigueur et une froideur quasi scientifiques, il en vient aussi à comparer le processus de vieillissement de ce dernier à un système électrique qui se détraquerait progressivement : " Court-circuits./ Petites pannes/ qui se multiplient rapidement.// Un coup dans le régulateur/ et il se venge/ Un choc électrique/ et c'est l'hécatombe » qui plonge «[q]uelques milliards de cellules/ dans l'obscurité.» Prise dans son ensemble et dans son histoire, l'espèce humaine se réduit finalement à « une minuscule pelote/ entre début et amnésie ${ }^{24}$. »

13 L'observation pour ainsi dire clinique de la déchéance physique, morale et intellectuelle qui caractérise la dernière partie de l'existence se double d'une méditation, somme toute traditionnelle, sur la brièveté et la fragilité de la vie. Là encore, ce sont d'abord les autres qui servent en quelque sorte de terrain d'expérimentation. Dans un poème intitulé Une moitié de la vie aussi, dont le titre pastiche le célèbre poème de Hölderlin, la disparition d'un entourage plus ou moins proche intervient sans même qu'on s'en rende vraiment compte :

Comme des ombres,

la troupe des absents grossit

si bien qu'on n'arrive plus à se souvenir

du couvre-chef de l'un, de la bouche de l'autre.

Et puis, un beau jour, arrive le moment

même si on ne le remarque pas, même s'il passe,

où tous ceux

qui vous ont nourri, aimé, instruit

et embrassé ont disparu pour la moitié d'entre eux ${ }^{25}$.

14 La mort des autres sert d'avertissement, puisqu'elle permet de mesurer le temps qui s'est écoulé et qu'elle renvoie à votre propre finitude. Mais ce sont aussi les choses qui nous rappellent notre condition de mortel. Le poème drolatique, Curriculum vitae, repose ainsi sur un parallèle, élucidé seulement à la fin, entre l'existence de l'être humain et la « vie » d'une chaussure entre le moment où elle quitte son carton et celui où elle le rejoint après avoir rempli de bons et loyaux services : «Elle finit donc là/ où nous finissons tous,/ dans une boîte sombre.// Ne subsista que l'âme,/ pour autant que

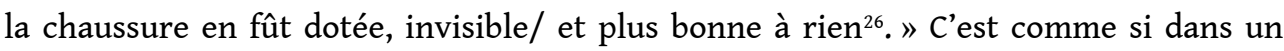
monde inexorablement réifié et où tout est réduit à une marchandise, l'objet, si ordinaire soit-il, mais perçu comme une somme d'expériences acquises, était investi du pouvoir de dire la signification profonde de l'homme, à moins qu'il ne s'agisse sous la plume de Enzensberger que d'une variation cocasse sur le thème éternel de la caducité humaine.

\section{Le temps des doutes et des interrogations}

15 On l'aura compris, la vieillesse est aussi le temps des doutes et des interrogations. Face à l'inéluctabilité de la mort, Enzensberger adopte une attitude complexe, faite 
d'angoisse, de sérénité et de dérision. Dans le poème First things first, le «je » qui, ici, se dissimule derrière un "nous" (pluriel de majesté ou de modestie ?) pratique volontiers la politique de l'autruche, prétextant d'autres priorités que les angoisses existentielles et repoussant même l'idée de la mort : "Commençons par jeter un coup d'œil/ dans l'agenda,// et puis on verra pour la suite ${ }^{27}$. " Dans d'autres textes en revanche, Enzensberger tente d'apprivoiser l'idée même de la mort. Revisitant la mythologie à l'aune du quotidien, il avoue ainsi fréquenter, simple "client » parmi d'autres "clients", riches ou pauvres, faibles ou puissants, un stand tenu par trois petites vieilles, réincarnation moderne et iconoclaste des divinités maîtresses de la destinée humaine de la naissance à la mort, et dédramatise de la sorte leur pouvoir : "Moi aussi, j'aime bien faire mes courses chez les Parques ${ }^{28}$." Il arrive même que le poète fasse ses adieux avant terme, la mort n'étant finalement appréhendée que comme une fin de partie/party venant sceller une vie assimilée à une fête pour laquelle le locuteur ne manque pas de remercier les participants :

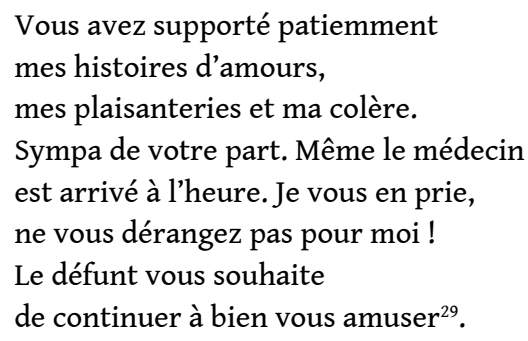

16 Si dans les vers cités supra, deuil et fête font bon ménage, il y a aussi des réticences et des résistances. Et c'est là qu'intervient contre toute attente, c'est-à-dire au vu des positions athées de l'auteur, la question de l'existence de Dieu. Dans le doute quant à la réalité du Très Haut, mieux vaut prendre ses précautions et lui écrire une lettre, comme le font les enfants s'adressant au père Noël, et se confondre en remerciements pour tous les plaisirs qu'il lui a/aurait apportés :

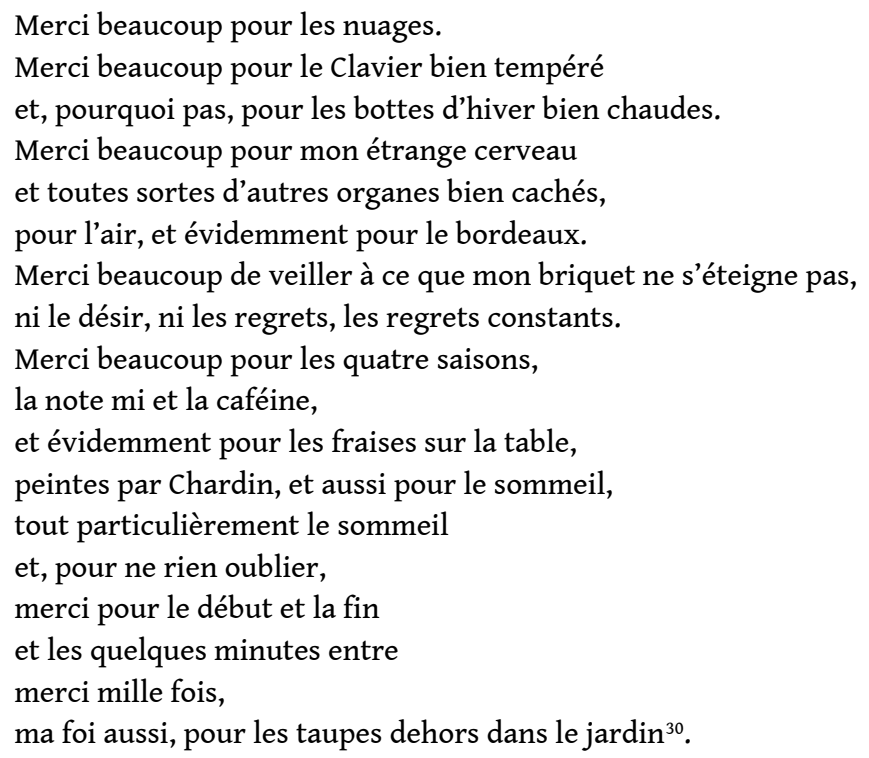

17 Cet adieu au monde constitue aussi un autoportrait de l'auteur qui en profite pour chanter son amour de la vie et faire l'inventaire de ses passions : se dessine l'image d'un épicurien, amateur de vin, de café et de tabac, d'un être raffiné sensible à la musique et à la peinture, mais tout aussi réceptif aux petits riens du quotidien, appréciant en somme les bons côtés de l'existence comme les mauvais dont font partie, entre autres, 
les taupes qui dévastent le jardin, puisque tout cela est l'essence même de la vie. Seul bémol : Dieu est-il là pour entendre les confessions du poète sur ses goûts et ses vices? Le titre du poème (Empfänger unbekannt - Retour à l'expéditeur), qui mêle l'allemand et le français (l'hypothétique destinataire serait-il polyglotte à l'instar de Enzensberger ou tout au moins bilingue ?), suggère tout de même que la lettre n'arrivera jamais à destination et que la communion du moribond risque de ne pas être entendue dans les hautes sphères. Toujours dans le doute, puisque le registre émotionnel semble être dans l'impasse, l'auteur n'hésite pas à recourir à la science, car il n'en va pas seulement de l'existence de Dieu, mais aussi et surtout de celle d'un audelà ou d'une vie après la mort. Comment trouver « l'étroite cachette,/ la tanière, la porte de derrière ${ }^{31}$ » ? À qui s'adresser, sinon aux spécialistes de la cosmologie, " grands prêtres » d'un monde devenu profane, «derniers mohicans/ de la métaphysique » pour savoir si « l'au-delà est un trou de ver/ et à combien d'univers parallèles [le moi] doit s'attendre ${ }^{32}$ »? L'auteur va même jusqu'à imaginer une théologie scientifique où règnerait un Dieu débordé par tout le travail qu'il doit fournir, théologie qui se révèle au bout du compte décevante, car elle ne débouche que sur l'idée de l'indifférence divine :

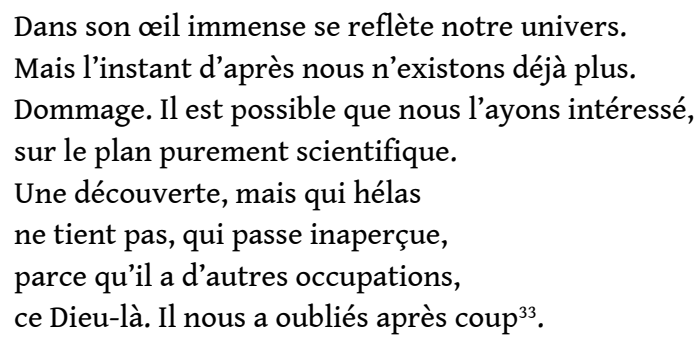

Que restera-t-il alors après la mort de ce moi «plus léger que l'air » qui n'intéresse même pas Dieu? «Bien des choses demeurent de toute façon/ en suspens./ Ce qui pèse peut-être le moins,/ c'est ce qui reste de nous,/ quand nous sommes sous la terre ${ }^{34}$. » Qu'abrite donc cette "enveloppe mortelle» que le poète ne cesse de sonder dans l'espoir d'en percer le mystère? Les réponses sont multiples :

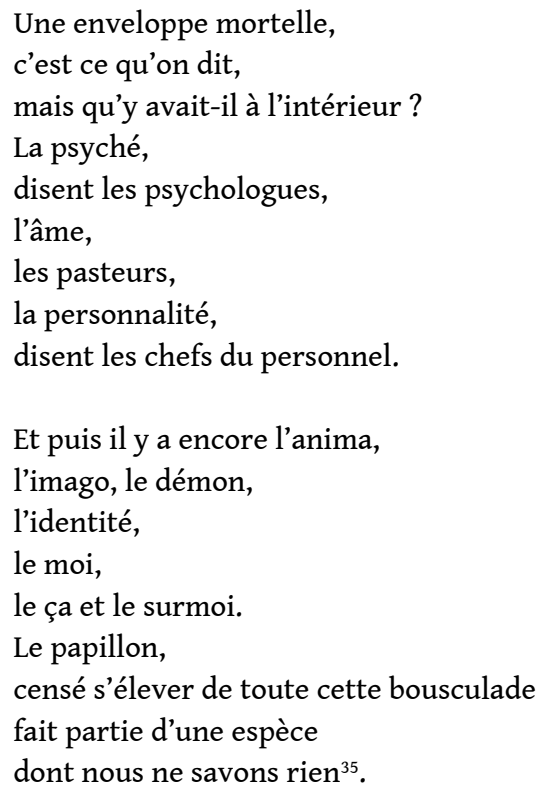

Peine perdue : ni la psychologie, ni la psychanalyse, ni la religion, ni la philosophie ni même le monde de l'entreprise, référence contemporaine insérée dans la somme des 
explications traditionnelles, ne sont en mesure de répondre à la question qui taraude le poète : qu'est-ce que l'homme et que reste-t-il de lui après la mort ? Certains textes inciteraient même à voir en Enzensberger non seulement un agnostique, mais aussi un nihiliste. Le poème intitulé Envoi dit crûment et avec une pointe de cynisme qu'il ne reste vraiment pas grand-chose de nous après la mort: «Et puis d'un seul coup nous n'étions plus là :/ Le numéro que vous avez demandé n'est plus en service./ Chacun d'entre nous a laissé à la postérité/ quelques vieilles factures et une brosse à dents ${ }^{36}$. " L'individu n'est donc rien une fois mort ou, après l'image de la vieille chaussure inutile, une fois de plus réduit à des objets, qui plus est d'une affligeante banalité. Enzensberger va même jusqu'à affirmer que l'être humain meurt une seconde fois, cette fois dans la mémoire des autres. Dans le poème R. I. P. (pour Requiescat in pace), le locuteur, qui se présente ici comme le porte-parole de l'espèce humaine, met en scène ses propres funérailles. Le défunt a beau tenter de nouer un dialogue par-delà la mort avec les vivants, rien n'y fait, on l'a déjà oublié, il n'est plus qu'un de ces personnages de ces albums à colorier, juste "une silhouette/ qui espère - comme ce serait bien! -/ que quelqu'un par un triste après-midi/ pluvieux/ réussisse à le colorier/ avec les crayons de couleur du souvenir ${ }^{37}$ ».

Est-on vraiment indispensable et surtout, à rebours du topos qui veut que l'œuvre soit garante d'immortalité, laisse-t-on vraiment une trace, tout poète que l'on soit ? C'est ce que thématisent deux poèmes dont le point commun est la forme dialogique. Dans le premier texte, le moi, visiblement atteint du syndrome de la page blanche, reçoit la visite d'un ange, qui plus est subalterne, pour s'entendre dire que franchement on se passe volontiers de lui et que "même l'ordinaire alisma [version personnelle du roseau de Pascal ?], si insignifiant fût-il,/ laisserait une trace " contrairement à lui. Contre toute attente, alors que le visiteur attend de l'autre qu'il s'insurge, ce dernier ne proteste pas: "Je ne bougeai pas. J'attendis/ qu'il soit parti, sans dire un $\operatorname{mot}^{38}$. " La lutte entre l'ange et l'homme qui fait la bête tourne court. Avec Osiris, le dieu des morts dans l'antiquité égyptienne, mais revu et corrigé, puisque devenu, technologie moderne oblige, l'acronyme de "Outer Space Intelligence Research and Investigation System », l'entretien tourne même au dialogue de sourds, voire au combat entre l'ombre et la lumière. La divinité psychopompe, impatiente de récupérer une âme qui refuse d'« accepter que la nuit soit la nuit» et de le suivre sous prétexte qu'elle a trop de choses à faire, qu'elle «ne veut pas aller se coucher. Par peur» et parce qu'elle «ne trouve pas le sommeil ", renvoie le candidat à la mort un brin réticent à son orgueil («Faux prétextes! D’autres étoiles obscures/ se font discrètes. Toi, tu veux juste// qu'on te voie. Que l'univers prenne note de toi. ») avant de l'humilier et de le mettre en face de son insignifiance : "Mais que tu te sois trouvé sur terre,/ fou électrique, croismoi,// personne ne l'a remarqué hormis moi ${ }^{39}$.» L'examen de passage est rude, puisqu'il confronte l'ancienne star de l'intelligentsia des années soixante, devenue au fil des ans une vénérable institution littéraire, à sa vanité au double sens de prétention et d'inanité.

Obscurité, légèreté/futilité et néant: telles sont donc les images récurrentes qui accompagnent la sénescence et contribuent à faire de la vieillesse moins un âge d'or mythologique que le temps de toutes les incertitudes.

Enzensberger n'a évidemment pas inventé la vieillesse, mais il construit sa propre image de la vieillesse à partir de celle des autres et de la sienne. Toutefois, même s'il pratique de plus en plus l'introspection, la vieillesse demeure un objet de curiosité 
intellectuelle qu'il observe avec une distance souvent teintée d'ironie. Fort de sa position en quelque sorte surplombante et de son immense culture générale, «le jeune homme » qui compte entre-temps quatre-vingt-deux printemps revisite les topoi littéraires, les méditations de l'âge classique sur la fuite du temps, la déchéance, le sens de la vie, la mort et la question d'une vie après la mort, moins du reste en vieux sage qu'en vieux singe impertinent. C'est de cette manière qu'il parvient à renouveler les lieux communs attachés à cet âge de la vie tout en s'inscrivant dans l'immense intertexte de la littérature universelle consacrée à la vieillesse. Ce faisant, il n'a de cesse de se moquer des stéréotypes et des formules toutes faites, n'hésitant pas à égratigner au passage les nouvelles figures du vieillir : ainsi en va-t-il de l'image positive du senior hyperactif régulièrement mise à la une des medias, mais casse-tête pour la gériatrie, qui lui arrache ce commentaire caustique: «Le corps vieillit, le cerveau rajeunit./ Contradiction tragique dont/ la science doit s'accommoder sans bougonner ${ }^{40}$ ». Finalement, la poésie de Hans Magnus Enzensberger ne propose pas une représentation close et achevée de la vieillesse, mais plutôt des impressions de vieillesse qui se contredisent souvent, tandis que lui-même multiplie les poses et les positions. Tour à tour angoissé ou serein, sérieux ou ironique, mélancolique ou bon vivant, le poète avance dans l'âge grâce à toute une série de masques. Tout se passe comme si cette période de l'existence, lorsqu'on est encore en vie, qui plus est toujours bon pied bon œil, ne pouvait se définir in fine que comme un work in progress, qui se construit et s'écrit au fil des jours ainsi qu'au gré des humeurs et... des lectures. C'est en ce sens qu'il faudrait sans doute nuancer le titre choisi pour cette contribution et parler au pluriel de portraits de l'artiste en vieillard. Le personnage demeure de fait insaisissable et on peut tout au mieux le cerner grâce à un autoportrait de Brentano que Enzensberger s'est du reste amusé à citer dans la thèse qu'il a consacrée à l'excentrique romantique : «Ma tête est la somme d'innombrables natures, j'y surprends souvent un public mêlét ${ }^{41}$ »

\section{Liste des œuvres citées}

Verteidigung der Wölfe (VW), Francfort-sur-le-Main, Suhrkamp, st 3047, 1999 (1957).

Landessprache (LS), Francfort-sur-le-Main, Suhrkamp, st 3047, 1999 (1960).

Blindenschrift (B), Francfort-sur-le-Main, Suhrkamp, st 3047, 1999 (1964).

Die Furie des Verschwindens (FW), Francfort-sur-le-Main, Suhrkamp, 1999 (1980).

Zukunftsmusik (Z), Francfort-sur-le-Main, Suhrkamp, st 3047, 1999 (1991).

Kiosk (K), Francfort-sur-le-Main, Suhrkamp, st 3047, 1999 (1995).

Leichter als Luft. Moralische Gedichte $(L L)$, Francfort-sur-le-Main, Suhrkamp, st 3666, 2005 (1999).

Die Geschichte der Wolken. 99 Meditationen (GW), Francfort-sur-le-Main, Suhrkamp, 2003. Rebus. Gedichte (R), Francfort-sur-le-Main, Suhrkamp, 2009. 


\section{NOTES}

1. Hans Magnus Enzensberger : Coda, in : R, p. 109. Cf. note 40 pour la traduction. Nous renvoyons ici à la liste des œuvres de Hans Magnus Enzensberger qui figure à la fin de l'article avec leurs abréviations respectives.

2. Alfred Andersch à propos du premier recueil de Hans Magnus Enzensberger, Verteidigung der Wölfe (1957) : «Endlich, endlich ist unter uns der zornige junge Mann erschienen [...]. », in : Reinhold Grimm (éd.) : Hans Magnus Enzensberger, Francfort-sur-le-Main, Suhrkamp, 1984, p. 62.

3. Die Scheintoten, in : LS, p. 18 : « und warten, verschieden, auf ihr Verscheiden.»

4. Dernières volontés, in : Hans Magnus Enzensberger : "Mausolée " précédé de "Défense des loups » et autres poésies. Traductions de Maurice Regnaut et Roger Pillaudin. Préface d'Hédi Kaddour, Paris, Gallimard, 2007, p. 22 ; Letztwillige Verfügung, in : VW, p. 32 : «Um die Auferstehung des Fleisches inzwischen und das ewige Leben/ werde ich mich, wenn es euch recht ist, selber bekümmern:/ Es ist meine Sache, nicht wahr? Lebt wohl.// Im Nachttisch sind noch ein paar Zigaretten. »

5. Compte à rebours, ibid., p. 110-111; Countdown, in : B, p. 48 : « am längsten dauert der Mensch:// solang/ bis jener dort in der Tiefe/ unsre Sekunden gezählt hat/ von zehn bis null. »

6. Ombrage, ibid., p. 140 ; Schattenwerk, in : B, p. 92 : «Schatten sind meine Werke».

7. Cf. Mausoleum. Siebenunddreißig Balladen aus der Geschichte des Fortschritts, Francfort-surle-Main, Suhrkamp, 1975.

8. Cf. Der Untergang der Titanic. Eine Komödie, Francfort-sur-le-Main, Suhrkamp, 1978.

9. « Dem Andenken meiner Eltern

Andreas und Eleonore

(1902-1990) (1905-2008)»

10. Restlicht, in : Z, p. 52 : « die von Minute zu Minute/ kleiner werdende Zeit ».

11. Jörg Lau: Hans Magnus Enzensberger. Ein öffentliches Leben, Francfort-sur-le-Main, Suhrkamp, st 3179, 2001 (1999), p. 362 : « Der Horizont wird enger. Die Zukunft wird nun gedacht als eine Zeit, die uns nicht gehört, nicht nach uns fragt, uns nicht zukommt. »

12. Coda, in : R, p. 109-110: «[...] Als ich zwanzig war, lange her,/ kam ich mir vor wie ein Toter, der keine Zukunft hat./ Jetzt ist sie da. [...]»

13. Altes Medium, in : K, p. 96 : «Was Sie vor Augen haben,/ meine Damen und Herren,/ dieses Gewimmel,/ das sind Buchstaben./ Entschuldigen Sie./ Entschuldigen Sie./ Schwer zu entziffern,/ ich weiß, ich weiß./ Eine Zumutung./ Sie hätten es lieber audiovisuell,/ digital und in Farben. »

14. Ibid., p. 96. On notera au passage l'emploi délibéré de l'anglicisme sous la plume d'Enzensberger qui n'ignore nullement le monde où il se meut, même s'il n'a de cesse de le fustiger.

15. Cf. Ins Lesebuch für die Oberstufe, in: VW, p. 88: "Lies keine Oden, mein Sohn, lies die Fahrpläne:/ sie sind genauer. Roll die Seekarten auf,/ eh es zu spät ist. Sei wachsam, sing nicht. »

16. Altes Medium, op. cit., p. 97 : «Ich wollte Ihnen nicht zu nahe treten./ Aber Sie wissen ja, wie das ist:/ Manche verlernen es nie.»

17. Der Geist des Vaters, in: K, p. 40: An manchen Abenden sitzt er da,/ wie früher, leicht gebückt,/ summend am Tisch/ unter der eisernen Lampe./ [...] Manchmal hört er mir zu,/ den schneeweißen Kopf geneigt,/ lächelt abwesend, zeichnet weiter/ an seinem wunderbaren Plan,/ den ich nicht begreifen kann,/ den er niemals vollenden wird./ Ich höre ihn summen. »

18. Eine zarte Regung, in : $L L$, p. 98 : "Mein Großvater,/ dieser Glückliche,/ verstand wenig vom Leben./ Er keuchte vor Appetit,/ trug flotte Hüte/ und glaubte häufig,/ er wäre im Recht./ Mit siebenundneunzig/ sah er, ungläubig/ und zum erstenmal,/ eine Klinik von innen./ 'Schade', murmelte er,/ 'hätte ich nur gewußt,/ wie reizend sie sind,/ die jungen Schwestern/ an meinem Bett,/ wie sanft ihre Hände,/ früher, viel früher/ wäre ich krank geworden',/ verzog die Mundwinkel,/ wandte die Augen/ zur Klingel und starb. » 
19. Herbst 1944, in : K, p. 22 : « wie sie hoch oben glitzerten/ am wolkenlosen Oktoberhimmel,/ die Bomberströme [...]// Sammeltassen und Engelshaar,/ Großvaters Pariser Postkarten/ (Oh là là) und sein Koppelschloß/ aus einem anderen Krieg, [...]// aber im Keller die Leichen/ sind immer noch da. »

20. Früher, in : $F W$, p. 60-61: «Die Vergangenheit, drückend/ und öde, ist unvorstellbar/ leicht entbehrlich. Heute noch/ weiß ich nicht genau,/ was das ist : Nostalgie. [...]// Packt doch die alten Fotos ein./ Ich verlasse mich lieber/ auf die Vergänglichkeit./ Sie läßt keine Rührung/ aufkommen, ist beharrlich/ und macht vor nichts halt. »

21. U-Bahn Wittenbergplatz, in : GW, p. 15 : «[...] abwärts/ in den alltäglichen Hades/ auf der Rolltreppe [...]// die waren doch auch einmal entflammt,/ früher, irgendwann, selbstvergessen,/ außer sich, strahlend/ vor Übermut, oder nicht?/ Wie kam es? Seit wann? Und warum?/ Draußen der Schnee ist auch schon wieder// zu Matsch geworden. »

22. Eingeständnis, in: LL, p. 103-104: "Immer schwerer gelingen mir/ Haß, Neid und Verachtung,/ diese jugendlichen Gefühle./ Ein Zeichen von Schwäche. »

23. Tae-Ho Kang: Poesie als Kritik und Selbstkritik. Hans Magnus Enzensbergers negative Poetik, Dissertation, Gesamthochschule Wuppertal, p. 226, note 858 : «Sogar könnte auch das Phänomen der "Gedankenflucht", das in seinen einigen Gedichten thematisiert wurde, eigentlich als eine Alterserscheinung verstanden werden, die er jedoch zur Form des dichterischen Sprechens macht. "

24. Limbisches System, in : Z, p. 98 : «Kurzschlüsse./ Kleine Defekte,/ die rasch eskalieren.// Ein Ruck in der Steuerung,/ und es nimmt Rache ;/ Ein elektrischer Stoß,/ und es läuft Amok.// Ein paar Milliarden Zellen/ im Dunkeln. Das Menschengeschlecht,/ ein winziges Knäuel/ zwischen Anfang und Amnesie. "

25. Auch eine Hälfte des Lebens, in: GW, p. 77 : «[...] Schattenhaft/ nahm der Trupp der Abwesenden zu,/ so daß du dich nicht mehr erinnern kannst,/ an die Mütze des einen, den Mund der andern./ Und dann, eines Tages, ist der Augenblick da,/ unbemerkt, und vergeht, an dem von denen,/ die dich gefüttert haben, gehaßt, belehrt/ und geküßt, die Hälfte verschwunden ist. »

26. Curriculum vitae, in : $L L$, p. 71 : «Also landete er, dort,/ wo wir alle landen,/ in einer dunklen Schachtel.// Übrig blieb nur die Seele,/ falls der Schuh eine hatte, unsichtbar/ und zu nichts zu gebrauchen. "

27. First things first, in : LL, p. 117 : "Erst einmal ein rascher Blick/ in den Terminkalender,// dann sehen wir weiter. »

28. Kiosk, in : K, p. 7 : « Auch ich kaufe gern/ bei den Parzen ein.»

29. Die Party, in : GW, p. 78 : « Geduldig habt ihr ertragen/ meine Liebesgeschichten,/ meine Witze und meine Wut./ Nett von euch. Auch der Notarzt/ war pünktlich zur Stelle. Bitte,/ laßt euch nicht stören!/ Gute Unterhaltung weiterhin/ wünscht der Verblichene. »

30. Empfänger unbekannt - Retour à l'expéditeur, in : K, p. 124 : «Vielen Dank für die Wolken./ Vielen Dank für das Wohltemperierte Klavier/ und, warum nicht, für die warmen Winterstiefel./ Vielen Dank für mein sonderbares Gehirn/ und für allerhand andre verborgne Organe,/ für die Luft, und natürlich für den Bordeaux./ Herzlichen Dank dafür, daß mir das Feuerzeug nicht ausgeht,/ und die Begierde, das Bedauern, das inständige Bedauern./ Vielen Dank für die vier Jahreszeiten,/ für die Zahl e und für das Koffein,/ und natürlich für die Erdbeeren auf dem Teller,/ gemalt von Chardin, sowie für den Schlaf,/ für den Schlaf ganz besonders,/ und, damit ich es nicht vergesse,/ für den Anfang und das Ende/ und die paar Minuten dazwischen/ inständigen Dank,/ meinetwegen für die Wühlmäuse draußen im Garten auch. »

31. Der Ausweg, in : GW, p. 31 : « die schmale heimliche Stelle,/ das Schlupfloch, die Hintertür. »

32. Fragen an die Kosmologen, in : GW, p. 118-119: «ob das Jenseits ein Wurmloch ist,/ und auf wie viele Paralleluniversen/ ich mich gefaßt machen muß?// [Ehrfürchtig lausche ich euern exakten Märchen,]/ ihr Hohepriester./ [So viele Fragen. An wen,/ wenn nicht an euch,]/ die letzten Mohikaner/ der Metaphysik,/ soll ich sie richten?» 
33. Wissenschaftliche Theologie, in : $L L$, p. 118-119 : «In seinem riesigen Auge/ spiegelt sich unser Universum./ Aber dann sind wir schon vorbei./ Schade. Womöglich hätten wir ihn,/ rein wissenschaftlich gesehen,/ interessiert. Eine Novität, nur leider/ nicht sehr haltbar, unbemerkt,/ weil er anderweitig beschäftigt war,/ dieser Gott. Er hat uns verschlafen. »

34. Leichter als Luft, in : LL, p. 38 : «Leichter als Luft [...]/ ist natürlich das Ich [...]// Vieles bleibt ohnehin/ in der Schwebe./ Am leichtesten wiegt vielleicht,/ was von uns übrigbleibt,/ wenn wir unter der Erde sind. »

35. Die Grablegung, in : K, p. 129 : «Eine sterbliche Hülle, so heißt es,/ aber was war drin?/ Die Psyche,/ sagen die Psychologen,/ die Seele,/ die Seelsorger,/ die Persönlichkeit,/ sagen die Personalchefs.// Dazu noch die Anima,/ die Imago, der Dämon,/ die Identität, das Ich,/ das Es und das Überich./ Der Schmetterling,/ der sich aus diesem Gedrängel/ erheben soll,/ gehört einer Art an,/ von der wir nichts wissen. »

36. Envoi, in : R, p. 80 : «Dann waren wir auf einmal verschwunden:/ Kein Anschluß unter dieser Nummer./ Jeder von uns hinterließ der Nachwelt/ ein paar alte Rechnungen und eine Zahnbürste. "

37. R. I. P., in : R, p. 101 : « [...] ein Umriß bloß,/ der darauf hofft - schön wär's! -,/ daß jemand an einem öden Nachmittag,/ wenn es draußen regnet,/ ihn ausmalen könnte/ mit den Buntstiften der Erinnerung. »

38. Die Visite, in: K, p. 118-119: "Sie können sich gar nicht vorstellen,/ sagte er,/ wie unentbehrlich Sie sind.// [...] Sogar der gemeine Froschlöffel, unscheinbar wie er ist,/ hinterließe eine Lücke, Sie nicht.// [...] Ich rührte mich nicht. Ich wartete,/ bis er verschwunden war, schweigend. »

39. Osiris (Outer Space Intelligence Research and Investigation System), in : K, p. 120-121 : « Sag, warum kannst du die Nacht/ nicht Nacht sein lassen?// [...] Weil ich nicht ins Bett will. Aus Angst./ Weil ich nicht einschlafen kann.// Ausreden! Andere Dunkelsterne/ schweigen diskret. Du willst ja nur,// daß man dich sieht. Das Universum/ soll Notiz von dir nehmen.// Aber daß du auf Erden warst,/ elektrischer Narr, glaub mir,// hat außer mir niemand bemerkt. »

40. Cf. note 1 .

41. Hans Magnus Enzensberger: Brentanos Poetik, Munich, dtv, 1973, p. 123 : « Mein Kopf ist eine Summe von vielerlei Naturen, in welchem ich oft ein sehr gemischtes Publikum ertappe.»

\section{RÉSUMÉS}

Entré dans l'histoire littéraire de la République Fédérale d'Allemagne sous l'étiquette du « jeune homme en colère », Hans Magnus Enzensberger compte entre-temps 82 printemps. Dès la fin des années 80 , la vieillesse entre dans son univers poétique et elle prendra une place de plus en plus importante dans les recueils des années 90 et 2000. Pour le poète vieillissant, c'est l'occasion de revisiter les thèmes traditionnellement attachés à cet âge de l'existence tels que la fuite du temps, la vanité de l'homme, la question du sens de la vie face à la mort inexorable et l'idée d'un au-delà, mais en se jouant de l'immense intertexte consacré à la vieillesse dans lequel il s'inscrit pourtant. Il en résulte moins une représentation achevée de la vieillesse qu'une mosaïque d'impressions fugitives et souvent contradictoires, comme si la vieillesse ne pouvait se définir conceptuellement, mais être tout au plus envisagée comme un work in progress qui se construit et s'écrit de jour en jour ainsi qu'au gré des humeurs et... des lectures. 
Hans Magnus Enzensberger who has gone down in the history of West German literature as « the angry young man » is 82 years old now. At the end of the 80 s, he showed an interest in the old age, which becomes more and more important in the poems of the $90 \mathrm{~s}$ and 2000s. For the poet who is getting old, it is the opportunity to renew life to the traditional themes about this period of life like the swift passage of time, the vanity of human existence, the questions about the meaning of life towards the inexorable death and afterlife by amusing himself with the literary tradition. The result is not a finished representation of old age, but a mosaic of contradictory impressions as if old age could not be conceptualized, but just described als a work in progress that is constructed and written with each passing day and according to the mood or the book.

Hans Magnus Enzensberger, der in die Literaturgeschichte der Bundesrepublik als »der zornige junge Mann« einging, ist inzwischen 82 Jahre alt geworden. Bereits Ende der 80er Jahre wächst sein Interesse an dem Altern, einem Thema, das in den Gedichtbänden der 90er und 2000er Jahre zunehmend an Bedeutung gewinnt. Dabei geht es dem alternden Dichter darum, die mit diesem Lebensalter traditionell verbundenen Themen - Vergänglichkeit, Hinfälligkeit des Lebens, Fragen nach dem Sinn des Lebens angesichts eines unabwendbaren Todes und nach einem Jenseits - neu zu bearbeiten und dabei mit der literarischen Tradition, als deren Mitglied er sich dennoch versteht, spielerisch umzugehen. Daraus ergibt sich nicht ein abgerundetes Bild vom Altern, sondern ein Mosaik von flüchtigen, oft widersprüchlichen Eindrücken, als ließe sich das Altern nicht begrifflich definieren, sondern höchstens als ein work in progress wahrnehmen, das lediglich von Tag zu Tag sowie je nach Laune (und nach Lektüre!) konstruiert und niedergeschrieben werden kann.

\section{INDEX}

Mots-clés : Allemagne, fin de vie, poésie, vieillesse

\section{AUTEUR \\ HÉLÈNE BOURSICAUT}

Université Rennes II 